\title{
Chronic Small Vessel Disease Affects Clinical Outcome in Patients with Acute Striatocapsular Stroke
}

\author{
V. Held K. Szabo H. Bäzner M.G. Hennerici \\ Department of Neurology, UniversitätsMedizin Mannheim, University of Heidelberg, Mannheim, Germany
}

\section{Key Words}

Aging $\cdot$ Outcome $\cdot$ Stroke $\cdot$ White matter

\begin{abstract}
Background: Recovery from stroke is presumed to be a function of a cerebral network. Chronic small vessel disease (SVD) has been shown to disrupt this network's integrity and has been proposed as a predictor of poor outcome after stroke. We studied this hypothesis in patients with acute ischemic stroke of the striatocapsular region, an area of pronounced cortical and subcortical connectivity. Methods: We identified 62 patients with isolated striatocapsular stroke from our stroke registry. The standardized workup included clinical rating according to the modified Rankin Scale (mRS) and MRI, rated according to the Fazekas scale for the extent of SVD, ranging from grade 0 to III. MRS at admission, at discharge, and a short-term recovery parameter (the difference between $\mathrm{mRS}$ at admission and discharge) were correlated with the extent of SVD. Comorbidity was assessed with the Charlson comorbidity index (CCl). Results: SVD was graded 0 in $7 \%, \mathrm{I}$ in $60 \%$, II in $18 \%$, and III in $16 \%$ of patients. The median $\mathrm{mRS}$ at discharge for the groups was 2, 1,2 and 4, and the median recovery parameter was $2,1,1$ and 0.5 , respectively. The extent of SVD significantly correlated with both the $\mathrm{mRS}$ at discharge and the recovery parameter. While age was also a significant predictor of these outcome parame-
\end{abstract}

ters, SVD severity was a significant predictor even after correction for age or CCI. Conclusions: SVD is a predictor of poor outcome and recovery in striatocapsular stroke, independent of age or comorbidity. Severe SVD disturbs the integrity of the cerebral network leading to aggravation of and poor recovery from neurological deficits.

Copyright $\odot 2011$ S. Karger AG, Basel

\section{Introduction}

With the advent of modern brain mapping techniques, neuroscience theories have shifted from functional localization in specific areas to functional integration, proposing a network of brain regions connected by anatomical tracts or functional association. Recovery from stroke is, presumably, a function of this widespread network and its ability to compensate for the damaged structures and to adapt to a given situation $[1,2]$. This ability to recover is apparently very different among individuals, as patients are known to recover from a stroke of similar location and size to very varying degrees [3]. Small vessel disease (SVD) with lesions in the white matter of the brain is a common finding in patients as well as healthy elderly individuals and has been implicated to play a prominent role in the disruption of the integrity of the cerebral network [4]. Based on these theories, SVD has been found to

\section{KARGER}

Fax +41613061234 E-Mail karger@karger.ch www.karger.com

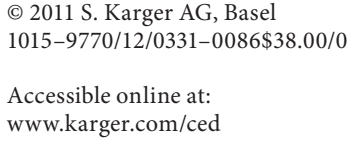

Valentin Held

Department of Neurology, UniversitätsMedizin Mannheim

Theodor-Kutzer-Ufer

DE-68137 Mannheim (Germany)

Tel. +49621 383 2885, E-Mail held@ @euro.ma.uni-heidelberg.de 
be a predictor of a negative outcome in stroke rather than stroke volume and topography, using isolated cerebellar infarction as a model [5].

To further test the hypothesis that the extent of SVD has an effect on functional recovery in acute stroke, the striatocapsular region seems to be appropriate for a couple of reasons. Firstly, the striatum is an integral part of several frontal-subcortical circuits as described by Alexander and Crutcher [6] and Alexander et al. [7, 8] and as such an area of particularly high connectivity involved in most of the higher cerebral functions. Secondly, deficits from striatocapsular stroke have been well described and commonly include a sensorimotor hemisyndrome but may also include a disturbance of speech and language or neuropsychological deficits [9]. In this study, we assessed the impact of the extent of SVD on the functional outcome after striatocapsular stroke.

\section{Materials and Methods}

Sixty-two patients (22 female, age range 23-91 years) with MRI-proven acute, isolated striatocapsular stroke were identified from a prospectively collected standardized stroke registry (out of 5,872 treated between November 2002 and February 2009). All patients were treated according to a standardized stroke unit protocol including cardiovascular and clinical monitoring by a neurologist, physiotherapy and speech therapy and standardized stroke care. All clinical data and technical investigations were recorded and documented according to a standardized acute stroke care protocol. The clinical status on admission was rated before thrombolysis (if applicable). In those patients requiring further inpatient rehabilitation therapy, the clinical outcome was taken from reports from the rehabilitation facility. Ethical approval for the collection and analysis of individuals' data was obtained according to appropriate national regulations. MRI was performed on a 1.5-tesla MR system (MAGNETOM Sonata, Siemens Medical Systems) on day 1-11 (mean day 1.5) after admission. A standardized stroke protocol was used in all patients including $\mathrm{T}_{2^{-}}$, $\mathrm{T}_{1}$-weighted, $\mathrm{T}_{2}$-weighted fluid-attenuated inversion recovery, time-of-flight magnetic resonance angiography, and diffusionweighted echo planar images.

Acute striatocapsular stroke was identified on diffusionweighted MRI as a hyperintense complete or partial lesion of the striatum (putamen and caudate nucleus, including the globus pallidus) and the internal capsule. Exclusion criteria were additional acute ischemic lesions outside the striatocapsular region, chronic territorial ischemic lesions as well as preexisting clinical neurological deficits. SVD in the deep white matter was identified on $\mathrm{T}_{2}$-weighted fluid-attenuated inversion recovery images and rated according to the visual scale set by Fazekas, ranging from 0 (no SVD) to 3 (confluent SVD) [10]. Rating was performed by a single rater blinded to relevant parameters (see fig. 1 for examples).

For statistical analysis, a short-term recovery parameter was defined as the difference between the modified Rankin Scale (mRS) at admission and discharge. For patients discharged to a

Chronic Small Vessel Disease and

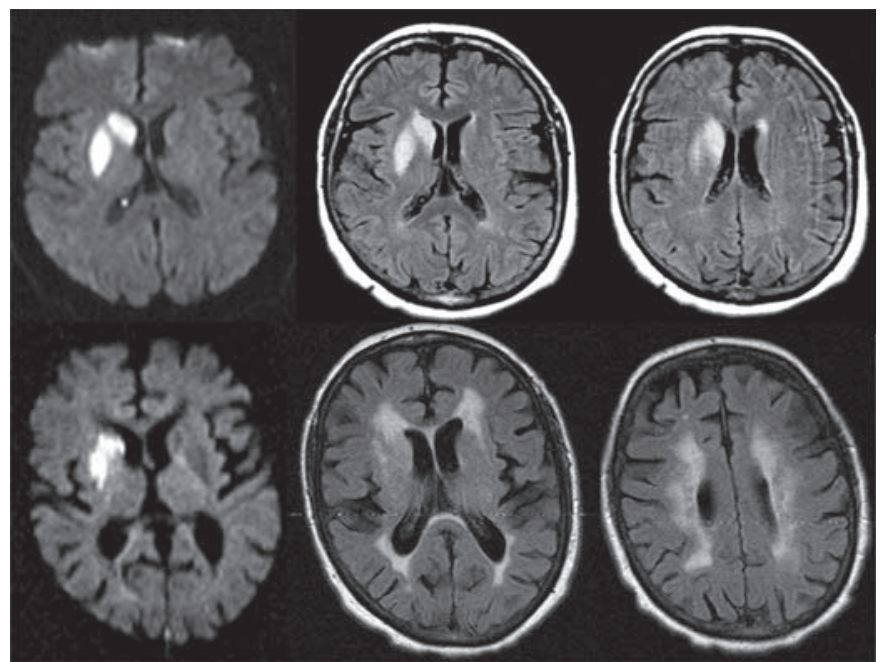

Fig. 1. Example of MRI findings in 2 acute striatocapsular stroke patients with SVD Fazekas grade I (upper row) and SVD Fazekas grade III (bottom row).

rehabilitation facility, their mRS at discharge from this facility was used. In all other cases the mRS at discharge from our department was used. The Charlson comorbidity index (CCI) was used to assess associated health problems [11]. It covers 30 diagnoses, weighted by their impact on 10 -year mortality. The items in the CCI were assessed to obtain a total comorbidity score to use in statistical analysis. Kendall's $\tau$ rank correlation was performed to evaluate the influence of SVD on stroke outcome. As a rank-based method it is suitable for nonparametric data and is robust [12]. To exclude confounding by age or CCI, Kendall's $\tau$ partial rank correlation was included in the analysis [13]. Fisher's exact test was performed to test for equal distribution among the groups. Calculations were performed using the $\mathrm{R}$ statistical package (The $\mathrm{R}$ Foundation for Statistical Computing, version 2.9.2).

\section{Results}

The most common symptoms leading to presentation were hemiparesis $(\mathrm{n}=59,95 \%)$, hemihypesthesia $(\mathrm{n}=34$, $56 \%)$, dysarthria $(\mathrm{n}=37,60 \%)$, and aphasia $(\mathrm{n}=21,34 \%)$. Median mRS on admission was 4 (3-5). The extent of white matter lesions was graded 0 in 4 , I in 37 , II in 11 , and III in 10 patients, respectively. Thirty-six of the 62 patients (58\%) were discharged to an inpatient rehabilitation facility, and 26 patients (42\%) could be discharged home requiring only ambulatory physiotherapy. The mean length of inpatient treatment was 9.1 days (range 3-18) for the first group and 54.7 days (range 15-106) for the second group. The median $\mathrm{mRS}$ at discharge when grouped by Fazekas grade 0-3 was 2 (1.75-2), 1 (1-3), 2 
Table 1. Patient data

\begin{tabular}{|c|c|c|c|c|c|}
\hline & Fazekas 0 & Fazekas I & Fazekas II & Fazekas III & $\mathrm{p}$ value \\
\hline Number & 4 & 37 & 11 & 10 & \\
\hline Female & $3(75 \%)$ & $14(38 \%)$ & $4(36 \%)$ & $5(50 \%)$ & $0.51^{1}$ \\
\hline Subsequent rehabilitation & $1(75 \%)$ & $20(54 \%)$ & $8(73 \%)$ & $7(70 \%)$ & $0.34^{1}$ \\
\hline Thrombolytic therapy & $2(50 \%)$ & $7(19 \%)$ & $0(0 \%)$ & $1(10 \%)$ & $0.11^{1}$ \\
\hline Mean age $\pm S D$, years & $61.5 \pm 26.7$ & $69.3 \pm 10.4$ & $78.7 \pm 5.9$ & $80.8 \pm 5.9$ & $0.0001^{2}$ \\
\hline CCI & $0.5(0-1.25)$ & $1(0-2)$ & $2(1-2.5)$ & $2(2-3.75)$ & $0.02^{2}$ \\
\hline Baseline mRS & $0(0-0.25)$ & $1(0-2)$ & $1(0-2)$ & $1(0.25-2)$ & $0.04^{2}$ \\
\hline mRS at admission & $4(2.75-5)$ & $4(3-5)$ & $3(2.5-4)$ & $4(3.25-4.75)$ & $0.37^{2}$ \\
\hline $\mathrm{mRS}$ at discharge & $2(1.75-2)$ & $1(1-3)$ & $2(2-3)$ & $4(2.25-4)$ & $0.02^{2}$ \\
\hline Difference of mRS & $2(0.75-3.25)$ & $1(1-2)$ & $1(0-1)$ & $0.5(0-1)$ & $0.004^{2}$ \\
\hline \multicolumn{6}{|l|}{ Risk factors } \\
\hline Arterial hypertension & $2(50 \%)$ & $30(81 \%)$ & $11(100 \%)$ & $10(100 \%)$ & $0.04^{1}$ \\
\hline Diabetes mellitus & $0(0 \%)$ & $9(22 \%)$ & $5(45 \%)$ & $5(50 \%)$ & $0.15^{1}$ \\
\hline Smoking & $1(25 \%)$ & $8(75 \%)$ & $0(0 \%)$ & $0(0 \%)$ & $0.15^{1}$ \\
\hline HLP & $2(50 \%)$ & $19(51 \%)$ & $3(27 \%)$ & $4(40 \%)$ & $0.56^{1}$ \\
\hline Hemiparesis & $4(100 \%)$ & $36(97 \%)$ & $9(82 \%)$ & $10(100 \%)$ & $0.25^{1}$ \\
\hline Aphasia & $2(50 \%)$ & $14(38 \%)$ & $3(27 \%)$ & $2(20 \%)$ & $0.63^{1}$ \\
\hline Dysarthria & $2(50 \%)$ & $25(68 \%)$ & $4(36 \%)$ & $6(60 \%)$ & $0.29^{1}$ \\
\hline \multicolumn{6}{|l|}{ Presumed stroke etiology } \\
\hline SVD & $1(25 \%)$ & $13(35 \%)$ & $8(73 \%)$ & $5(50 \%)$ & $0.13^{1}$ \\
\hline Cardioembolic & $2(50 \%)$ & $10(27 \%)$ & $1(9 \%)$ & $3(30 \%)$ & $0.37^{1}$ \\
\hline Large artery disease & $1(25 \%)$ & $10(27 \%)$ & $1(9 \%)$ & $0(0 \%)$ & $0.18^{1}$ \\
\hline Other/unknown & $0(0 \%)$ & $4(11 \%)$ & $1(9 \%)$ & $2(20 \%)$ & $0.83^{1}$ \\
\hline
\end{tabular}

Figures in parentheses are ranges or percentages as indicated. HLP = Hyperlipoproteinemia.

${ }^{1}$ Calculated by Fisher's exact test. ${ }^{2}$ Calculated by Kendall's $\tau$.

(2-3) and 4 (2.25-4), respectively, and the median recovery parameter, defined as the difference between $\mathrm{mRS}$ on admission and discharge, was 2 (0.75-3.25), 1 (1-2), 1 (01) and $0.5(0-1)$, respectively. Table 1 provides an overview of patient characteristics by the extent of SVD.

The median $\mathrm{mRS}$ on admission was not significantly related to the extent of SVD, CCI or age; however, the extent of SVD showed a significant correlation with age $(\tau=0.387, \mathrm{p}$ value $<0.001)$ and CCI $(\tau=0.26, \mathrm{p}$ value $=$ $0.017)$. The extent of SVD predicted negative outcome in terms of $\mathrm{mRS}$ at discharge $(\tau=0.245, \mathrm{p}$ value $=0.02)$ and the recovery parameter $(\tau=-0.315, \mathrm{p}$ value $=0.004)$. These relations largely persisted after correcting for age ( $\tau=0.13$ and -0.25 , p value $=0.13$ and 0.005 , respectively), the CCI $(\tau=0.20$ and -0.29 , p value $=0.03$ and $<0.001$, respectively), or $\mathrm{mRS}$ at baseline, before the index stroke ( $\tau=0.21$ and 0.30 , p value 0.02 and $<0.001$, respectively).

Figures 2 and 3 illustrate this relationship. While none of the patients with Fazekas grade 0 required care for daily needs, more than two thirds of the patients with Fazekas grade III did. Furthermore, 20 out of 41 (49\%) pa- tients with no or little SVD (Fazekas grade 0 or I) improved significantly (difference of $m R S \geq 2$ ), while only 4 out of 21 (19\%) patients with relevant SVD (Fazekas grade II or III) improved significantly, and 3 (14\%) even showed functional deterioration during treatment (fig. 3).

Age was also a predictor of a negative outcome in terms of final $\mathrm{mRS}(\tau=0.339$, $\mathrm{p}$ value $<0.001)$ and less so of development during treatment $(\tau=-0.247$, $\mathrm{p}$ value $=$ $0.01)$. Similarly, this relationship was still significant after correction for Fazekas score for the final $\mathrm{mRS}(\tau=0.27$, $\mathrm{p}$ value $=0.002)$ but not for the difference in $\operatorname{mRS}(\tau=$ -0.14 , $\mathrm{p}$ value $=0.10$ )

CCI predicted a negative outcome $(\tau=0.24$, $\mathrm{p}$ value $=$ 0.02 ) but not a difference in functional status.

Patients who went to a rehabilitation center after treatment in the acute hospital had a significantly worse status at discharge (median mRS at discharge 3 vs. $1, \mathrm{p}$ value $<0.001)$ and a trend towards a smaller success of rehabilitation (median difference of $\mathrm{mRS} 1$ vs. 1.5 , $\mathrm{p}$ value $=$ 0.08). This might be explained by the finding that patients with a good primary outcome were in no need of further 


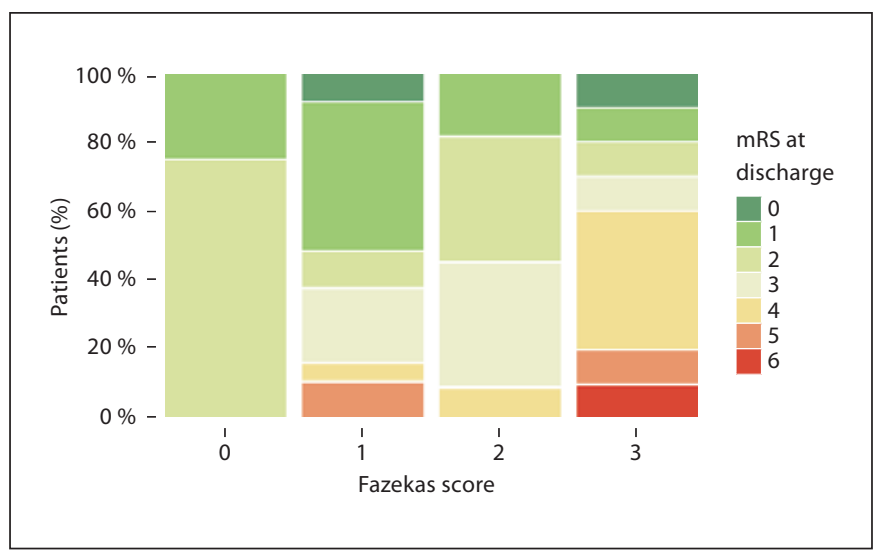

Fig. 2. Frequency distribution of the mRS score in patients with different degrees of SVD according to the Fazekas score.

rehabilitation and patients who had not recovered well primarily were unlikely to do so later. SVD was equally prevalent in patients who underwent rehabilitation as in those who did not ( $\mathrm{p}$ value $=0.34$ ).

Other established risk factors, namely diabetes mellitus, smoking, hyperlipoproteinemia and male sex, did not predict a negative outcome either in terms of functional status on discharge or in terms of development during treatment. Arterial hypertension was related to a worse outcome in terms of mRS on discharge ( $p$ value $=$ 0.01 ) but not in terms of course during treatment. While there were trends towards more patients with acute ischemia of presumed microangiopathic cause in patients with more pronounced SVD, and more acute stroke due to cardioembolism or large artery disease in patients with less pronounced SVD, these trends did not reach statistical significance. Similarly, patients with less severe SVD tended to receive thrombolytic therapy more often than patients with severer SVD; however, this trend was not statistically significant.

\section{Discussion}

In this study, we have shown that in patients with isolated striatocapsular stroke, $\mathrm{mRS}$ at discharge and shortterm recovery, as measured by the difference between $\mathrm{mRS}$ on admission and discharge, depend on the extent of SVD. Of the 10 patients who recovered the most (by 3 or 4 points on the mRS), none had more than mild SVD (Fazekas grade 0 or I), while all patients whose status de-

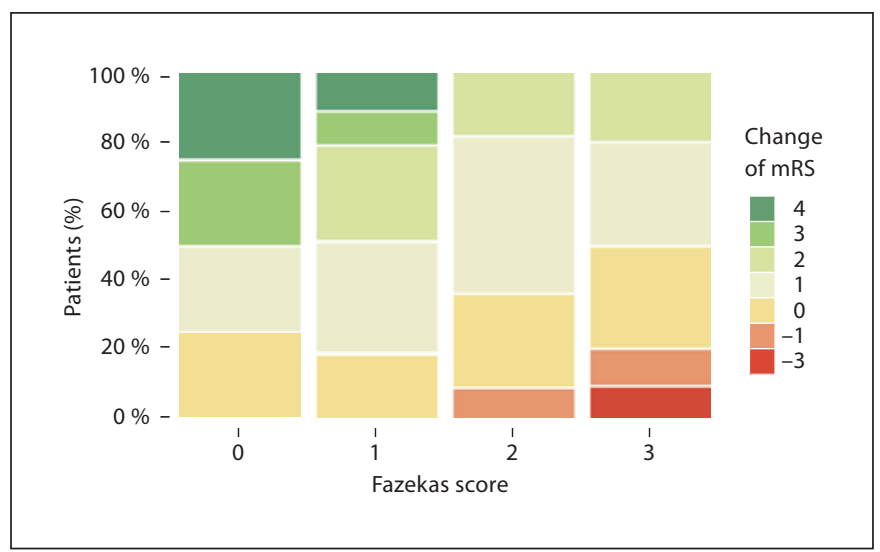

Fig. 3. Frequency distribution of the short-term outcome parameter in patients with different degrees of SVD according to the Fazekas score.

teriorated during treatment (by 1-3 points on the mRS) had moderate or severe SVD (Fazekas grade II or III).

Interestingly, the connection between SVD and the 2 measures of outcome and age, respectively, is inverse. While age is a stronger predictor of poor final status than SVD, SVD is a stronger predictor of poor recovery during treatment than age. This may be explained by a nonsignificant trend toward a better functional status on admission in patients with more SVD, or indeed be a sign of age being a cause of lower functional status in general versus SVD being the cause of poor recovery after stroke.

Recent research has addressed the relationship between SVD and functional outcome after stroke. In a first study on patients with isolated cerebellar infarction, functional outcome correlated with the coexistence of age-related white matter lesions rather than stroke volume and topography [5]. Arsava et al. [14] and Ay et al. [15] extended these findings to supratentorial stroke and were able to show that this influence is also independent of other factors, including age, initial deficit, and infarct volume growth. In 2009, Kissela et al. [16] found that severe periventricular white matter disease was significantly associated with poor functional outcome at 3 months. In a recently published study, extensive SVD was associated with severe Glasgow Coma Scale scores at admission, 30-day mortality, and the relative risk for mortality in patients with intracerebral hemorrhage [17]. In an even more detailed approach, Liou et al. [18] evaluated the impact of periventricular SVD and subcortical SVD in patients with a Fazekas score $>2$ on post-stroke 
functional outcomes. The authors found an unfavorable functional stroke at 30 days in patients with periventricular but not subcortical white matter lesions supporting the different roles of different white matter locations. However, not all studies have confirmed these findings: in 75 first-ever middle cerebral artery strokes, the presence of white matter lesions on MRI did not predict independence in activities of daily living 1 year after the stroke [19]. Contradictory results also came from several CT-based studies [20-22], which could not detect this relationship, probably due to the low sensitivity of CT scans to detect SVD.

Ay et al. [15] demonstrated that SVD increases susceptibility to infarct growth and thus have provided a first mechanism explaining how SVD may cause poor recovery from stroke. However, the same group has also demonstrated that this effect is at least partially independent of infarct growth [14]. Based on previous studies indicating that SVD causes a disruption of the cerebral network, we hypothesized that this disruption is the cause of poor recovery. Both the cerebellum and the striatum are areas of exceptional connectivity and take central positions in the circuits that control higher cerebral functions. One important difference in our study population compared to all others is the fact that we selected patients with similar infarct patterns as opposed to analyzing a heterogeneous group with ischemic stroke affecting different regions of the brain.

This study has several limitations. (1) Visual rating scales, such as the Fazekas scale, have been argued not to be very precise and have low interrater reliability [23]. (2) The interval between admission and discharge is highly various between patients and, overall, follow-up is short, thus the data only allow a conclusion on short-term functional outcome. (3) The difference in mRS between admission and discharge provides a way to illustrate a gain of abilities and independence during stroke rehabilitation which goes beyond functional status at a given point and, as it is calculated at an individual level, beyond correcting for status at admission. The mRS is, however, an ordinal scale and values should not ordinarily be added or subtracted. With this caveat in mind, we still believe that interesting information can be drawn from its use. (4) In limiting stroke location to the striatocapsular region we have chosen an area of high connectivity and a well-defined, functionally relevant deficit, seemingly ideal to test the hypothesis that SVD, via a disturbance of the cerebral network, hinders stroke recovery. However, this also limits the extent to which our results can be generalized to other stroke locations. Since striatocapsular stroke is a rather rare type of ischemia, this also makes for a seemingly limited number of patients in our study. In this respect, this study is in line with other studies, which have studied stroke in this location and have reported on similar or smaller numbers of patients [9, 24-26]. We cannot exclude that our results have at least been partially influenced by varying degrees of cerebral atrophy in our patients. On the other hand, the causes of cerebral atrophy are manifold and there is some evidence that SVD may at least be one of these causes [27]. So trying to correct for atrophy may actually hide some of the veritable effects of SVD. Nevertheless, the interactions of age, SVD, cerebral atrophy and stroke outcome are interesting and should be further investigated in the future.

While we believe that this and previous studies have demonstrated the effect of SVD in recovery from stroke, several aspects remain unclear. Knowledge about the interaction of location and mechanism of stroke and the locations of SVD is still limited. All studies in this field, so far, have used scales of dependence and disability, especially the Barthel Index and mRS, for assessing the clinical relevance. It remains unclear which precise deficits cause the decline of functional status. The question still remains whether SVD hinders stroke recovery or whether stroke aggravates compensated deficits from SVD. More differentiated measures of outcome, such as task-specific motor skill tests, may yield important information. Modern functional MRI may point a way to further investigate network integrity and function in stroke and SVD [28].

\section{References}

1 Carmichael ST: Plasticity of cortical projections after stroke. Neuroscientist 2003;9:6475.

2 Di Filippo M, Tozzi A, Costa C, Belcastro V, Tantucci M, Picconi B, Calabresi P: Plasticity and repair in the post-ischemic brain. Neuropharmacology 2008;55:353-362.

\footnotetext{
3 Jørgensen $H$, Nakayama $H$, Raaschou $H$, Vive-Larsen J, Støier M, Olsen T: Outcome and time course of recovery in stroke. I. Outcome. The Copenhagen Stroke Study. Arch Phys Med Rehabil 1995;76:399-405.

-4 Ryberg C, Rostrup E, Sjöstrand K, Paulson O, Barkhof F, Scheltens P, van Straaten E,
}

Fazekas F, Schmidt R, Erkinjuntti T, Wahlund LO, Basile A, Pantoni L, Inzitari D, Waldemar G, LADIS study group: White matter changes contribute to corpus callosum atrophy in the elderly: the LADIS study. AJNR Am J Neuroradiol 2008;29:14981504. 
5 Grips E, Sedlaczek O, Bäzner H, Fritzinger M, Daffertshofer M, Hennerici M: Supratentorial age-related white matter changes predict outcome in cerebellar stroke. Stroke 2005;36:1988-1993.

-6 Alexander G, Crutcher M: Functional architecture of basal ganglia circuits: neural substrates of parallel processing. Trends Neurosci 1990;13:266-271.

7 Alexander G, Crutcher M, DeLong M: Basal ganglia-thalamocortical circuits: parallel substrates for motor, 'prefrontal' and 'limbic' functions. Prog Brain Res 1990;85:119-146.

-8 Alexander G, DeLong M, Strick P: Parallel organization of functionally segregated circuits linking basal ganglia and cortex. Annu Rev Neurosci 1986;9:357-381.

-9 Donnan GA, Bladin PF, Berkovic SF, Longley WA, Saling MM: The stroke syndrome of striatocapsular infarction. Brain 1991;114: 51-70.

-10 Fazekas F, Chawluk J, Alavi A, Hurtig H, Zimmerman R: MR signal abnormalities at 1.5 T in Alzheimer's dementia and normal aging. AJR American J Roentgenol 1987;149: 351-356.

$\checkmark 11$ Charlson M, Pompei P, Ales K, MacKenzie C: A new method of classifying prognostic comorbidity in longitudinal studies: development and validation. J Chronic Dis 1987;40: 373-383.

12 Kendall M: A new measure of rank correlation. Biometrika 1938;30:81-93.

13 Kendall M: Partial rank correlation. Biometrika 1942;32:277-283.
14 Arsava E, Rahman R, Rosand J, Lu J, Smith EE, Rost NS, Singhal A, Lev MH, Furie K, Koroshetz W, Sorensen A, Ay H: Severity of leukoaraiosis correlates with clinical outcome after ischemic stroke. Neurology 2009; 72:1403-1410.

15 Ay H, Arsava E, Rosand J, Furie K, Singhal A, Schaefer P, Wu O, Gonzalez R, Koroshetz W, Sorensen A: Severity of leukoaraiosis and susceptibility to infarct growth in acute stroke. Stroke 2008;39:1409-1413.

16 Kissela B, Lindsell CJ, Kleindorfer D, Alwell K, Moomaw CJ, Woo D, Flaherty ML, Air E, Broderick J, Tsevat J: Clinical prediction of functional outcome after ischemic stroke: the surprising importance of periventricular white matter disease and race. Stroke 2009; 40:530-536.

17 Lee SH, Kim BJ, Ryu WS, Kim CK, Kim N, Park BJ, Yoon BW: White matter lesions and poor outcome after intracerebral hemorrhage: a nationwide cohort study. Neurology 2010;74:1502-1510.

18 Liou LM, Chen CF, Guo YC, Cheng HL, Lee HL, Hsu JS, Lin RT, Lin HF: Cerebral white matter hyperintensities predict functional stroke outcome. Cerebrovasc Dis 2010;29: 22-27.

19 Schiemanck SK, Kwakkel G, Post MWM, Kappelle LJ, Prevo AJH: Predicting longterm independency in activities of daily living after middle cerebral artery stroke: does information from MRI have added predictive value compared with clinical information? Stroke 2006;37:1050-1054.

20 Jørgensen H, Nakayama H, Raaschou H, Olsen T: Leukoaraiosis in stroke patients. The Copenhagen stroke study. Stroke 1995;26 588-592.
21 Hénon H, Godefroy O, Leys D, Mounier-Vehier F, Lucas C, Rondepierre P, Duhamel A, Pruvo JP: Early predictors of death and disability after acute cerebral ischemic event. Stroke 1995;26:392-398.

22 Wiszniewska M, Devuyst G, Bogousslavsky J, Ghika J, Van Melle G: What is the significance of leukoaraiosis in patients with acute ischemic stroke? Arch Neurol 2000;57:967973.

-23 Scheltens P, Erkinjuntti T, Leys D, Wahlund L, Inzitari D, del Ser T, Pasquier F, Barkhof F, Mäntylä R, Bowler J, Wallin A, Ghika J, Fazekas F, Pantoni L: White matter changes on CT and MRI: an overview of visual rating scales. European task force on age-related white matter changes. Eur Neurol 1998;39: 80-89.

24 Lee KB, Oh HG, Roh H, Ahn MY: Can we discriminate stroke mechanisms by analyzing the infarct patterns in the striatocapsular region? Eur Neurol 2008;60:79-84.

$>25$ Jung S, Hwang SH, Lee BC: Distinct clinical expressions of striatocapsular infarction according to cortical manifestations. Eur J Neurol 2004;11:627-633.

-26 Nicolai A, Lazzarino LG, Biasutti E: Large striatocapsular infarcts: clinical features and risk factors. J Neurol 1996;243:44-50.

-27 Nitkunan A, Lanfranconi S, Charlton RA, Barrick TR, Markus HS: Brain atrophy and cerebral small vessel disease: a prospective follow-up study. Stroke 2010;42:133-138.

$>28$ Chen K, Reiman EM, Huan Z, Caselli RJ, Bandy D, Ayutyanont N, Alexander GE: Linking functional and structural brain images with multivariate network analyses: a novel application of the partial least square method. Neuroimage 2009;47:602-610. 